\title{
Quantum-memory-assisted entropic uncertainty principle under noise
}

\author{
Z. Y. Xu ${ }^{1},{ }^{*}$ W. L. Yang ${ }^{2}$, and M. Feng ${ }^{2 \dagger}$ \\ ${ }^{1}$ School of Physical Science and Technology, Soochow University, Suzhou 215006, China \\ ${ }^{2}$ State Key Laboratory of Magnetic Resonance and Atomic and Molecular Physics, \\ Wuhan Institute of Physics and Mathematics, Chinese Academy of Sciences, \\ and Wuhan National Laboratory for Optoelectronics, Wuhan 430071, China
}

\begin{abstract}
The measurement outcomes of two incompatible observables on a particle can be precisely predicted when it is maximally entangled with a quantum memory, as quantified recently [Nature Phys. 6, 659 (2010)]. We explore the behavior of the uncertainty relation under the influence of local unital and nonunital noisy channels. While the unital noises only increase the amount of uncertainty, the amplitude-damping nonunital noises may amazingly reduce the amount of uncertainty in the longtime limit. This counterintuitive phenomenon could be justified by different competitive mechanisms between quantum correlations and the minimal missing information after local measurement.
\end{abstract}

PACS numbers: 03.65.Ta, 05.40.Ca, 03.65.Yz

One of the most remarkable features of quantum mechanics is the restriction of our ability to simultaneously predict the measurement outcomes of two incompatible observables with certainty, which is called Heisenberg's uncertainty principle [1]. Nowadays, the more modern approach to characterize the uncertainty principle is the use of entropic measures rather than with standard deviations [2]. If we denote the probability of the outcome $x$ by $p(x)$ when a given quantum state $\rho$ is measured by an observable $X$, the Shannon entropy $H(X)=-\sum_{x} p(x) \log _{2} p(x)$ characterizes the amount of uncertainty about $X$ before we learn its measurement outcomes [3]. For two non-commuting observables $Q$ and $R$, the entropic uncertainty relation can be expressed as $H(Q)+H(R) \geq \log _{2} \frac{1}{c}[2]$, where $c=\max _{\alpha, \beta}\left|\left\langle\phi_{\alpha} \mid \varphi_{\beta}\right\rangle\right|^{2}$ with $\left|\phi_{\alpha}\right\rangle$ and $\left|\varphi_{\beta}\right\rangle$ the eigenstates of $Q$ and $R$, respectively. Since $c$ is independent of the states of system to be measured, the widely studied entropic uncertainty relation provides us with a more general framework of quantifying uncertainty than the standard deviations (See a review in [4]).

However, the entropic uncertainty relation may be violated if a particle is initially entangled with another one [5]. In the extreme case, an observer holding the particle $A$, maximally entangled with particle $B$ (quantum memory), is able to precisely predict the outcomes of two incompatible observables $Q$ and $R$ acting on $A$. A stronger entropic uncertainty relation was conjectured by Renes and Boileau [6], and later proved by Berta et al. [7]

$$
S(Q \mid B)+S(R \mid B) \geq \log _{2} \frac{1}{c}+S(A \mid B),
$$

where $S(A \mid B)=S\left(\rho_{A B}\right)-S\left(\rho_{B}\right)$ is the conditional von Neumann entropy with $S(\rho)=-\operatorname{tr}\left(\rho \log _{2} \rho\right)$ the von Neumann entropy [3]. $S(X \mid B)$ with $X \in(Q, R)$ is the conditional von Neumann entropy of the post-measurement state $\rho_{X B}=\sum_{x}\left(\left|\psi_{x}\right\rangle\left\langle\psi_{x}\right| \otimes \mathbb{1}\right) \rho_{A B}\left(\left|\psi_{x}\right\rangle\left\langle\psi_{x}\right| \otimes \mathbb{1}\right)$ after quantum system $A$ is measured by $X$, where $\left\{\left|\psi_{x}\right\rangle\right\}$ are the eigenstates of the observable $X$ and $\mathbb{1}$ is the identity operator. Although the proof of this quantum-memoryassisted entropic uncertainty relation is rather complex, the meaning is clear: the entanglement of systems $A$ and $B$ may lead to a negative conditional entropy $S(A \mid B)$ [8], which will in turn beat the lower bound $\log _{2} \frac{1}{c}$. Especially when $A$ and $B$ are maximally entangled, the simultaneous measurement of $Q$ and $R$ can be precisely predicted $[7,9]$. In recent, two parallel experiments $[10,11]$ have confirmed the quantum-memory-assisted entropic uncertainty relation.

Quantum objects are inevitably in contact with environments and a consequence of the interaction is decoherence or dissipation [3, 12]. So several questions naturally arise: How environmental noises influence the quantummemory-assisted entropic uncertainty principle? Will the noisy channels surely and only increase the amount of uncertainty because of disentanglement? Is quantum correlation the only key factor for this uncertainty principle under noise? To answer these questions, we consider in this Letter two categories of noises: unital and nonunital noisy channels. Intuition tells us that the uncertainty will increase due to the noise-induced disentanglement. We demonstrate that it is true for local unital noises, but may fail for a local amplitude-damping noise, a typical nonunital channel, in the long-time limit. The mechanism behind this counterintuitive phenomenon is explored. As a by-product, we present quantum correlation witness under specific noises by the new uncertainty relation.

We focus on the uncertainty game model illustrated in Ref. [7]: Bob sends qubit $A$, initially entangled with another qubit $B$ (quantum memory), to Alice. Then, Alice measures either $Q$ or $R$, and announces her measurement choice to Bob. Eq. (1) captures Bob's uncertainty about Alice's measurement outcome. We assume the two-qubit system to be initially prepared in a class of states with the maximally mixed subsystems $\left[\rho_{A(B)}=\mathbb{1}^{A(B)} / 2\right][13]$

$$
\rho_{A B}=\frac{1}{4}\left(\mathbb{1}^{A} \otimes \mathbb{1}^{B}+\sum_{j=1}^{3} C_{\sigma_{j}} \sigma_{j}^{A} \otimes \sigma_{j}^{B}\right),
$$


where $\sigma_{j}$ with $j \in\{1,2,3\}$ are the standard Pauli matrices, and the coefficients $C_{\sigma_{j}}=\operatorname{tr}_{A B}\left(\rho_{A B} \sigma_{j}^{A} \otimes\right.$ $\sigma_{j}^{B}$ ) satisfy $0 \leq\left|C_{\sigma_{j}}\right| \leq 1$. The state of this type is also called Bell-diagonal state, because it can be diagonalized as a convex combination of four Bell states: $\rho_{A B}=\lambda_{\Phi^{+}}\left|\Phi^{+}\right\rangle\left\langle\Phi^{+}\left|+\lambda_{\Phi^{-}}\right| \Phi^{-}\right\rangle\left\langle\Phi^{-}\right|+$ $\lambda_{\Psi^{+}}\left|\Psi^{+}\right\rangle\left\langle\Psi^{+}\left|+\lambda_{\Psi^{-}}\right| \Psi^{-}\right\rangle\left\langle\Psi^{-}\right|$, with eigenstates $\left|\Phi^{ \pm}\right\rangle=$ $(|00\rangle \pm|11\rangle) / \sqrt{2}$ and $\left|\Psi^{ \pm}\right\rangle=(|01\rangle \pm|10\rangle) / \sqrt{2}$, and corresponding eigenvalues $\lambda_{\Phi^{ \pm}}=\left(1 \pm C_{\sigma_{1}} \mp C_{\sigma_{2}}+C_{\sigma_{3}}\right) / 4$ and $\lambda_{\Psi^{ \pm}}=\left(1 \pm C_{\sigma_{1}} \pm C_{\sigma_{2}}-C_{\sigma_{3}}\right) / 4$, respectively. Considering the positivity requirement $\lambda_{\Phi^{ \pm}}, \lambda_{\Psi^{ \pm}} \geq 0$, all Bell-diagonal states should be confined geometrically within a tetrahedron in a three-dimensional space spanned by $\left(C_{\sigma_{1}}, C_{\sigma_{2}}, C_{\sigma_{3}}\right)$ [13] (See Fig. 1), providing an intuitive geometric picture for exploring the quantummemory-assisted entropic uncertainty principle.

Before investigating the noise effect, we first consider how to consistently reach the lower bound of Eq. (1). We employ the set of Pauli observables $\left\{\sigma_{j}\right\}$ with $j \in$ $\{1,2,3\}$. The conditional von Neumann entropy after qubit $A$ was measured by one of the Pauli observables can be expressed as $S\left(\sigma_{j} \mid B\right)=H_{b i n}\left(\frac{1+C_{\sigma_{j}}}{2}\right)$, where $H_{b i n}(p)=-p \log _{2} p-(1-p) \log _{2}(1-p)$ is the binary entropy [3]. Therefore, if we choose two of the Pauli observables $Q=\sigma_{j}$ and $R=\sigma_{k}(j \neq k)$ for measurement, the left-hand side of Eq. (1) can be written as

$$
U=H_{b i n}\left(\frac{1+C_{\sigma_{j}}}{2}\right)+H_{b i n}\left(\frac{1+C_{\sigma_{k}}}{2}\right) .
$$

On the other hand, the complementarity $c$ of the observables $\sigma_{j}$ and $\sigma_{k}$ is always equal to $1 / 2$ and the reduced density matrix of the Bell-diagonal state is a maximally mixed state, i.e., $S\left(\rho_{B}\right)=1$. Therefore, the right-hand side of Eq. (1) reduces to $S\left(\rho_{A B}\right)$ and takes the form

$$
U_{b}=-\sum_{\chi=\Phi, \Psi ; \varepsilon= \pm} \lambda_{\chi^{\varepsilon}} \log _{2} \lambda_{\chi^{\varepsilon}}
$$

In general, Eq. (4) provides a lower bound of uncertainty of Eq. (3). Having in mind the choice of our measurements $\sigma_{j}$ and $\sigma_{k}$, we may check that if the initial Bell-diagonal state meets the following condition

$$
C_{\sigma_{i}}=-C_{\sigma_{j}} \cdot C_{\sigma_{k}},(i \neq j \neq k),
$$

$U \equiv U_{b}$ will be strictly satisfied in Eq. (1), implying a direct measurement of the degree of uncertainty by the joint entropy $S\left(\rho_{A B}\right)$ of the whole system. In what follows, we name Eq. (5) as the state preparation and measurement choice (SPMC) condition for this new entropic uncertainty principle.

We assume that qubit $A$ will experience a local noisy channel when sent to Alice, but qubit $B$ is a quantum memory free from noise [14]. The evolved state of the whole system can be characterized by the quantum map

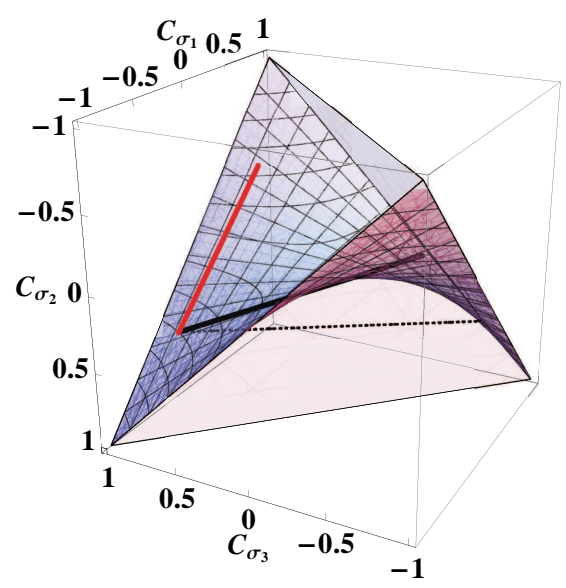

FIG. 1: (Color online) The geometry of Bell-diagonal states with the blue (gray) tetrahedron representing the set of all Bell-diagonal states, where the meshed surface means the valid Bell diagonal states meeting the SPMC condition $C_{\sigma_{2}}=-C_{\sigma_{1}} \cdot C_{\sigma_{3}}$. The black solid, red (gray) solid, and black dotted lines represent trajectories of Bell-diagonal states $\left(C_{\sigma_{1}}, C_{\sigma_{2}}, C_{\sigma_{3}}\right)=(-0.5,0.4,0.8)$ under local bit-flip, phase-flip, and bit-phase-flip noises, respectively.

$\mathcal{M}\left(\rho_{A B}\right)=\sum_{\mu}\left(\kappa_{\mu} \otimes \mathbb{1}\right) \rho_{A B}\left(\kappa_{\mu} \otimes \mathbb{1}\right)^{\dagger}$ with $\left\{\kappa_{\mu}\right\}$ the local Kraus operators satisfying $\sum_{\mu} \kappa_{\mu}^{\dagger} \kappa_{\mu}=\mathbb{1}$.

We first consider several paradigmatic types of local unital noisy channels: bit-flip, bit-phase-flip, and phaseflip (equivalent to phase damping), satisfying the unital condition: $\Lambda_{u}^{A}\left(\frac{1}{2} \mathbb{1}^{A}\right)=\frac{1}{2} \mathbb{1}^{A}[15]$, with $\Lambda_{u}^{A}\left(\rho_{A}\right)=$ $\sum_{\mu} \kappa_{\mu} \rho_{A} \kappa_{\mu}^{\dagger}$. The corresponding Kraus operators are denoted by $\kappa_{0}^{l}=\sqrt{1-\eta_{l}} \mathbb{1}, \kappa_{1}^{l}=\sqrt{\eta_{l}} \sigma_{l}$ with $l=1,2,3$ representing bit-flip, bit-phase-flip, and phase-flip channels respectively (in the following, bit-flip, bit-phase-flip, and phase-flip noises are also called $\Sigma_{1}, \Sigma_{2}, \Sigma_{3}$ noises, respectively), and $\eta_{l}$ represents the probability of the noise taking place. It is convenient to check that the state of qubits $A$ and $B$ initially prepared in a Bell-diagonal state $\left(C_{\sigma_{1}}, C_{\sigma_{2}}, C_{\sigma_{3}}\right)$ will still be of a Bell-diagonal type when passing through one of the three noisy channels: $\mathcal{M}\left(\rho_{A B}\right)=\sum_{\chi=\Phi, \Psi ; \varepsilon= \pm} \lambda_{\chi^{\varepsilon}}^{\prime}\left|\chi^{\varepsilon}\right\rangle\left\langle\chi^{\varepsilon}\right|$, where $\lambda_{\Phi^{ \pm}}^{\prime}=[1 \pm$ $\left.C_{\sigma_{1}}^{\prime} \mp C_{\sigma_{2}}^{\prime}+C_{\sigma_{3}}^{\prime}\right] / 4$ and $\lambda_{\Psi^{ \pm}}^{\prime}=\left[1 \pm C_{\sigma_{1}}^{\prime} \pm C_{\sigma_{2}}^{\prime}-C_{\sigma_{3}}^{\prime}\right] / 4$ [16] with the three parameters

$$
\begin{aligned}
C_{\sigma_{l}}^{\prime} & =C_{\sigma_{l}}, \\
C_{\sigma_{m}}^{\prime} & =\left(1-2 \eta_{l}\right) C_{\sigma_{m}},(m \neq l) .
\end{aligned}
$$

Here $l=1,2,3$ represent qubit $A$ suffering from $\Sigma_{1}, \Sigma_{2}, \Sigma_{3}$ noisy channels, respectively. Assuming qubit $A$ will experience one of the above noises, one can find that for quantum states initially prepared in Belldiagonal states meeting the SPMC condition $C_{\sigma_{i}}=$ $-C_{\sigma_{j}} \cdot C_{\sigma_{k}}$, the quantum-memory-assisted entropic uncertainty of the observables $\sigma_{j}$ and $\sigma_{k}$ can consistently reach the lower bound, if no $\Sigma_{i}$ noise takes place [17]. 


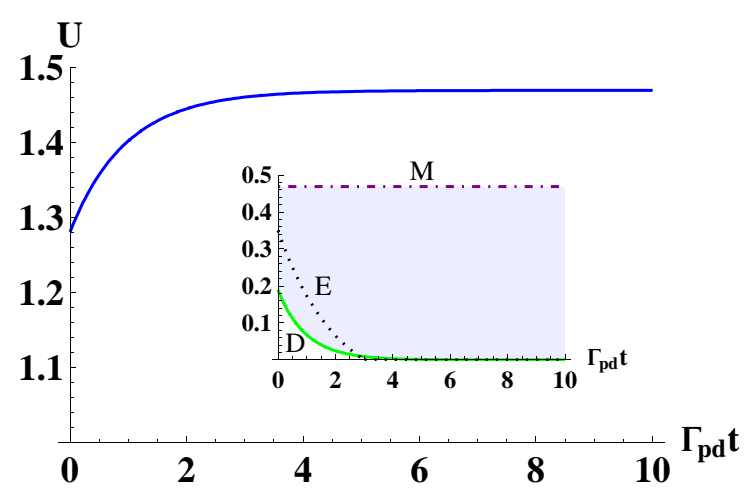

FIG. 2: (Color online) Uncertainty of the observables $\sigma_{1}$ and $\sigma_{3}$ with initial state $\left(C_{\sigma_{1}}, C_{\sigma_{2}}, C_{\sigma_{3}}\right)=(-0.5,0.4,0.8)$ under the local phase-damping channel with $\Gamma_{p d}$ the damping rate. The inset: entanglement (E), discord (D), and minimal missing information about $A$ after $B$ is measured (M) versus $\Gamma_{p d} t$.

For an illustration, the geometric picture of Belldiagonal states satisfying the SPMC condition $C_{\sigma_{2}}=$ $-C_{\sigma_{1}} \cdot C_{\sigma_{3}}$ is depicted as the meshed surface in Fig. 1. On this surface, the uncertainty of the observables $\sigma_{1}$ and $\sigma_{3}$ is equal to $S\left(\rho_{A B}\right)$. The black solid, red (gray) solid, and black dotted lines represent, respectively, trajectories of the Bell-diagonal state, initially prepared in $\left(C_{\sigma_{1}}, C_{\sigma_{2}}, C_{\sigma_{3}}\right)=(-0.5,0.4,0.8)$, under $\Sigma_{1}, \Sigma_{3}$, and $\Sigma_{2}$ noises. Apparently, $\Sigma_{1}$ and $\Sigma_{3}$ noises will not break the SPMC condition, since their trajectories are always on the surface, whereas $\Sigma_{2}$ noise, due to the departure from the surface, will definitely break the SPMC condition (except for two points). As a result, the SPMC condition will be consistently satisfied if no $\Sigma_{2}$ noise takes place.

To explore the influence of quantum correlations on this uncertainty relation, we relate the lower bound of Eq. (1) to the definition of discord (a measure for quantum correlations): $D=-S(A \mid B)+\min _{\left\{B_{k}\right\}} \sum_{k} q_{k} S\left(\rho_{A}^{k}\right)[18]$, where $\min _{\left\{B_{k}\right\}} \sum_{k} q_{k} S\left(\rho_{A}^{k}\right)$ (denoted by $M$ in the following) captures the minimal missing information about $A$ after $B$ is measured, and $\rho_{A}^{k}=\operatorname{tr}_{B}\left\{B_{k} \rho_{A B} B_{k}^{\dagger}\right\} / q_{k}$ is the resulting state after the complete measurement $\left\{\mathrm{B}_{k}\right\}$ on qubit $B$, and $q_{k}=\operatorname{tr}_{A B}\left\{B_{k} \rho_{A B} B_{k}^{\dagger}\right\}$. Therefore, we have

$$
U \geq \log _{2} \frac{1}{c}+M-D
$$

For Bell-diagonal states, $M$ can be expressed as [19]

$$
M=H_{b i n}\left(\frac{1+C_{\max }}{2}\right)
$$

with $C_{\max }=\max \left\{\left|C_{\sigma_{1}}^{\prime}\right|,\left|C_{\sigma_{2}}^{\prime}\right|,\left|C_{\sigma_{3}}^{\prime}\right|\right\}$. According to Eq. (6), if $\Sigma_{i}$ noise takes place, as long as $\left|C_{\sigma_{i}}\right| \geq\left|C_{\sigma_{j}}\right|,\left|C_{\sigma_{k}}\right|$, we may have $M=H_{b i n}\left(\frac{1+\left|C_{\sigma_{i}}\right|}{2}\right)$, which is a constant, and implies that the uncertainty is fully dependent on the quantum correlations between qubit $A$ and quantum memory $B$. Especially, if initial state is prepared according to SPMC condition $C_{\sigma_{j}}=-C_{\sigma_{i}} \cdot C_{\sigma_{k}}$ (or $C_{\sigma_{k}}=-C_{\sigma_{i}} \cdot C_{\sigma_{j}}$ ), the equality in Eq. (7) can be consistently satisfied, which suggests that measuring the uncertainty of the observables $\sigma_{i}$ and $\sigma_{k}$ (or $\sigma_{i}$ and $\sigma_{j}$ ) can be directly employed to witness quantum correlations

$$
D=\text { const }-U \text {, }
$$

with const $=\log _{2} \frac{1}{c}+H_{b i n}\left(\frac{1+\left|C_{\sigma_{i}}\right|}{2}\right)$ a constant.

As an example, we consider the phase-damping channel with the Kraus operators $\kappa_{0}^{p d}=|0\rangle\left\langle 0\left|+e^{-\frac{\Gamma p d^{t}}{2}}\right| 1\right\rangle\langle 1|$ and $\kappa_{1}^{p d}=\sqrt{1-e^{-\Gamma_{p d} t}}|1\rangle\langle 1|$, which is equivalent to the phase-flip channel with $\eta_{3}=\left(1-e^{-\frac{\Gamma_{p d} t}{2}}\right) / 2$ [3]. Qubit $A$ and quantum memory $B$ are initially prepared in a Bell-diagonal state $\left(C_{\sigma_{1}}, C_{\sigma_{2}}, C_{\sigma_{3}}\right)=(-0.5,0.4,0.8)$ satisfying the SPMC condition $C_{\sigma_{2}}=-C_{\sigma_{1}} \cdot C_{\sigma_{3}}$, then qubit $A$ sent through the phase-damping channel will not break the SPMC condition, and the uncertainty of the observables $\sigma_{1}$ and $\sigma_{3}$ can be directly used to detect quantum correlations $D=$ const $-U$, with const $=1+H_{b i n}(0.9)$ and $U=H_{b i n}(0.9)+H_{b i n}\left(0.5-0.25 e^{-\Gamma_{p d} t / 2}\right)$. As shown in Fig. 2, the uncertainty will increase in the long-time limit due to the gradually missing quantum correlations.

The dynamics of discord and entanglement (measured by concurrence [20]) are shown in the inset of Fig. 2 . As we know, entanglement will not increase under local noisy channels [21]. In addition, since $\mathcal{M}\left(\rho_{A B}\right)=$ $\sum_{\chi=\Phi, \Psi ; \varepsilon= \pm} \lambda_{\chi^{\varepsilon}}^{\prime}\left|\chi^{\varepsilon}\right\rangle\left\langle\chi^{\varepsilon}\right|$ holds for bit-flip, bit-phase-flip, and phase-flip (phase-damping) noises, we may conclude that these unital channels are also semiclassical according to the definition presented in Ref. [15]. Therefore, quantum correlations never increase under above local unital channels [15]. However, are we sure that the decrease of quantum correlations including entanglement and discord make the outcomes of two incompatible observables more uncertain?

To further explore this problem, in what follows, we consider a nonunital and nonsemiclassical local channel, i.e., the amplitude-damping noise with Kraus operators $\kappa_{0}^{a d}=e^{-\frac{\Gamma_{a d} t}{2}}|0\rangle\langle 0|+| 1\rangle\left\langle 1\left|, \kappa_{1}^{a d}=\sqrt{1-e^{-\Gamma_{a d} t}}\right| 1\right\rangle\langle 0|$ $[3,22]$. Here $\Lambda_{n u}^{A}\left(\frac{1}{2} \mathbb{1}^{A}\right)=\left[e^{-\Gamma_{a d} t}|0\rangle\langle 0|+(2-\right.$ $\left.\left.e^{-\Gamma_{a d} t}\right)|1\rangle\langle 1|\right] / 2$ is not maximally mixed, which implies that the state through a noisy channel to be measured by Alice is not a Bell-diagonal state, and the SPMC condition presented above is not satisfied anymore. We may only study the lower bound of uncertainty instead. Provided the initial condition $\left|C_{\sigma_{1}}\right| \geq\left|C_{\sigma_{2}}\right|, M$ can be expressed as

$$
M=\min \left\{M_{x}, M_{z}\right\},
$$

where $M_{x}=H_{b i n}\left(\frac{1+u}{2}\right)$ with $u=$
$\sqrt{e^{-\Gamma_{a d} t}\left[C_{\sigma_{1}}^{2}+2 \cosh \left(\Gamma_{a d} t\right)-2\right]}$ and $M_{z}=$ 


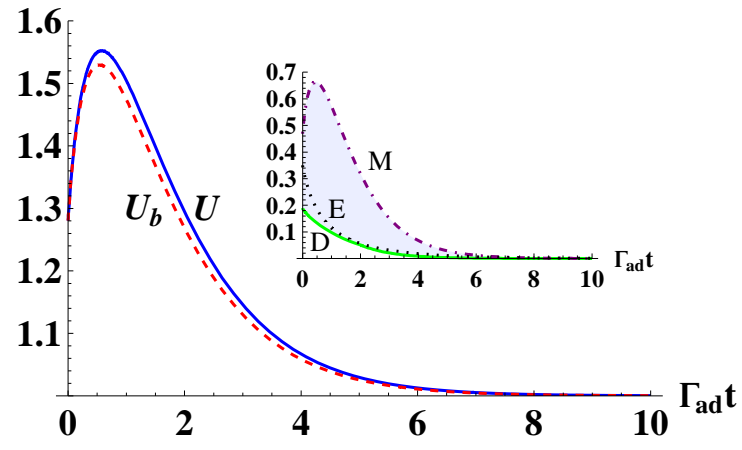

FIG. 3: (Color online) $U$ and $U_{b}$ of the observables $\sigma_{1}$ and $\sigma_{3}$ with initial state $\left(C_{\sigma_{1}}, C_{\sigma_{2}}, C_{\sigma_{3}}\right)=(-0.5,0.4,0.8)$ under the local amplitude-damping channel with $\Gamma_{a d}$ the damping rate. The inset: same parameters as in Fig. 2.

$\frac{H_{b i n}\left(v_{+}\right)+H_{b i n}\left(v_{-}\right)}{2}$ with $v_{ \pm}=\left(1 \pm C_{\sigma_{3}}\right) \exp \left(-\Gamma_{a d} t\right) / 2$ [23], which is time-dependent and may also be nonmonotonic. Therefore, in contrast to Eq. (9), the uncertainty relation here cannot be directly employed to detect quantum correlations. Nevertheless, Fig. 3 demonstrates a counterintuitive phenomenon: the uncertainty of two incompatible observables might be reduced under the influence of the amplitude-damping noise in the long-time limit (for a general discussion see in [23]). The key factor for the uncertainty reduction should not be the quantum correlations, which are decreasing in this case. Therefore, the quantum correlation is not the only decisive factor for the amount of the uncertainty.

Now we summarize the above phenomena: (i) The uncertainty (or lower bound) will increase under local unital noisy channels while it may be reduced under the nonunital noise channel. (ii) The relation between quantum correlations and uncertainty is subtle, since the reduced uncertainty happens in the case that quantum correlations, including discord and entanglement are also reduced under above local amplitude-damping channel.

The first phenomenon can be explained mathematically. We assume the bipartite system is initially prepared with the maximally mixed subsystems. Therefore, in general, we have $U_{b}=\log _{2} \frac{1}{c}+S(A \mid B)=$ $\log _{2} \frac{1}{c}+S\left(\rho_{A B}\right)-\log _{2} d_{B}$ (d $d_{B}$ the dimension of quantum memory $B)$. As the joint entropy will not decrease under local unital channels: $S\left[\mathcal{M}_{l u}\left(\rho_{A B}\right)\right] \geq S\left(\rho_{A B}\right), U_{b}$ will increase under local unital noise [23], however, it may not be true for local nonunital channels.

In order to understand the physical origin of above phenomena, especially the second one, we reconsider the treatment in Eq. (7). Apparently, the uncertainty is related to the discrepancy between $M$ and $D$, not just the quantum correlations only, and it is decided by the competition between quantum correlations and the minimal missing information of single particle after local measurement on another one. For illustration, the competition between $M$ and $D$ are depicted with blue (gray) shades in the insets of Figs. 2 and 3. Quantum correlations, including entanglement and discord, will decrease in both cases. However, the most difference is that Eq. (10) is not a monotonic function, which may even decrease under amplitude-damping channel. That is to say, the missing information by local measurements may be reduced in the long-time limit, which in turn lowers the uncertainty.

The work presented above can be immediately investigated by all-optical setups, where the state preparation and measurement can be realized like in Refs. [10, 11] and the noisy channels can be simulated according to Refs. [24, 25].

Before ending this Letter, we mention some open problems waiting for solution: What is the physical mechanism behind the competition between $\mathrm{M}$ and $\mathrm{D}$ in a general frame? Will this counterintuitive phenomenon exist in all nonunital noisy channels and for all quantum states? Is it possible to find the minimum uncertainty achievable in the presence of nonunital noise? Can we find similar phenomena by other entropy measures, e.g., the uncertainty relation based on smooth entropy [26]? Most fascinatingly, is it possible to directly utilize the decoherence or dissipation properties illustrated in this Letter to perform quantum information tasks such as quantum key distribution?

In summary, we have studied the noise effect on quantum-memory-assisted entropic uncertainty principle. By investigating different noises, we have demonstrated that local unital noises will surely increase the uncertainty, but under the influence of a nonunital amplitude-damping channel, we found remarkably that the uncertainty might even be reduced. Our work is the first step toward the study of the noise effect on the quantum-memory-assisted entropic uncertainty principle and can be practically estimated by quantum tomography with currently experimental setups in $[10,11,24,25]$.

This work is supported by the National Natural Science Foundation of China under Grant No. 10974225 and No. 11004226, and by the National Fundamental Research Program of China under Grant No. 2012CB922102. Z.Y.X. also acknowledges support by Soochow University Startup Fund under Grant No. Q410800911.

Note added: After finishing this work, we became aware of several topics on the role of quantum correlations in the entropic uncertainty principle, such as arXiv:1202.0939 and arXiv:1203.3153.

* Electronic address: zhenyuxu@suda.edu.cn

$\dagger$ Electronic address: mangfeng@wipm.ac.cn

[1] W. Heisenberg, Z. Phys. 43, 172 (1927).

[2] I. Bialynicki-Birula and J. Mycielski, Commun. Math. 
Phys. 44, 129 (1975); D. Deutsch, Phys. Rev. Lett. 50, 631 (1983); K. Kraus, Phys. Rev. D 35, 3070 (1987); H. Maassen and J. B. M. Uffink, Phys. Rev. Lett. 60, 1103 (1988)

[3] M. A. Nielsen and I. L. Chuang, Quantum Computation and Quantum Information (Cambridge University Press, Cambridge, England, 2000).

[4] S. Wehner and A. Winter, New J. Phys. 12, 025009 (2010).

[5] Y.-K. Kim and Y. Shih, Found. Phys. 29, 1849 (1999); H. F. Hofmann and S. Takeuchi, Phys. Rev. A 68, 032103 (2003).

[6] J. M. Renes and J.-C. Boileau, Phys. Rev. Lett. 103, 020402 (2009).

[7] M. Berta, M. Christandl, R. Colbeck, J. M. Renes, and R. Renner, Nature Phys. 6, 659 (2010).

[8] I. Devetak and A. Winter, Proc. R. Soc. A 461, 207 (2005).

[9] A. Winter, Nature Phys. 6, 640 (2010).

[10] C.-F. Li, J.-S. Xu, X.-Y. Xu, K. Li, and G.-C. Guo, Nature Phys. 7, 752 (2011).

[11] R. Prevedel, D. R. Hamel, R. Colbeck, K. Fisher, and K. J. Resch, Nature Phys. 7, 757 (2011).

[12] C. W. Gardiner and P. Zoller, Quantum Noise (SpringerVerlag, Berlin, 2004); H.-P. Breuer and F. Petruccione, The Theory of Open Quantum Systems (Oxford University Press, Oxford, 2007).

[13] R. Horodecki and M. Horodecki, Phys. Rev. A 54, 1838 (1996).

[14] B. Julsgaard, J. Sherson, J. I. Cirac, J. Fiurasek, E. S. Polzik, Nature (London) 432, 482 (2004).

[15] A. Streltsov, H. Kampermann, and D. Bruß, Phys. Rev. Lett. 107, 170502 (2011).

[16] J. Maziero, L. C. Céleri, R. M. Serra, and V. Vedral, Phys. Rev. A 80, 044102 (2009); L. Mazzola, J. Piilo, and S. Maniscalco, Phys. Rev. Lett. 104, 200401 (2010); M. D. Lang and C. M. Caves, Phys. Rev. Lett. 105, 150501 (2010).

[17] Given $\Sigma_{i}$ noise takes place, we may have $C_{\sigma_{i}}^{\prime}=C_{\sigma_{i}}$, $C_{\sigma_{j}}^{\prime}=\left(1-2 \eta_{i}\right) C_{\sigma_{j}}$, and $C_{\sigma_{k}}^{\prime}=\left(1-2 \eta_{i}\right) C_{\sigma_{k}}$ according to Eq. (6). Assuming that the SPMC condition can still be consistently satisfied under $\Sigma_{i}$ noise, i.e., $C_{\sigma_{i}}^{\prime}=-C_{\sigma_{j}}^{\prime}$. $C_{\sigma_{k}}^{\prime}$, we may get the relation $C_{\sigma_{i}}=-C_{\sigma_{j}} \cdot C_{\sigma_{k}} \cdot\left(1-2 \eta_{i}\right)^{2}$, which requires $\left(1-2 \eta_{i}\right)^{2}=1$, i.e., $\eta_{i}=0$ or 1 (just two extreme cases).

[18] H. Ollivier and W. H. Zurek, Phys. Rev. Lett. 88, 017901 (2001); L. Henderson and V. Vedral, J. Phys. A 34, 6899 (2001)

[19] S. Luo, Phys. Rev. A 77, 042303 (2008).

[20] W. K. Wootters, Phys. Rev. Lett. 802245 (1998).

[21] R. Horodecki, P. Horodecki, M. Horodecki, and K. Horodecki, Rev. Mod. Phys. 81, 865 (2009).

[22] T. Yu and J. H. Eberly, Phys. Rev. Lett. 93140404 (2004).

[23] See details in Supplemental Material.

[24] M. P. Almeida, F. de Melo, M. Hor-Meyll, A. Salles, S. P. Walborn, P. H. Souto Ribeiro, L. Davidovich, Science 316, 579 (2007).

[25] J.-S. Xu, X.-Y. Xu, C.-F. Li, C.-J. Zhang, X.-B. Zou, and G.-C. Guo, Nature Commun. 1, 7 (2010).

[26] M. Tomamichel and R. Renner, Phys. Rev. Lett. 106, 110506 (2011).

\section{Supplemental Material}

\section{The SPMC condition of Eq. (5)}

For clarity, we denote Eq. (4) by $U_{b}=$ $-\sum_{x, y=0,1} \lambda_{x y} \log _{2} \lambda_{x y}$, with $\lambda_{x y}=\left[1+(-1)^{x} C_{\sigma_{1}}-\right.$ $\left.(-1)^{x+y} C_{\sigma_{2}}+(-1)^{y} C_{\sigma_{3}}\right] / 4$ the eigenvalues of a Belldiagonal state. We first consider one of the SPMC conditions, e.g., $C_{\sigma_{1}}=-C_{\sigma_{2}} \cdot C_{\sigma_{3}}$. Then $\lambda_{x y}=$ $\left[1-(-1)^{x+y} C_{\sigma_{2}}\right] \cdot\left[1+(-1)^{y} C_{\sigma_{3}}\right] / 4$ and

$$
\begin{aligned}
& U_{b}=-\sum_{x, y=0,1}\left\{\frac{\left[1-(-1)^{x+y} C_{\sigma_{2}}\right] \cdot\left[1+(-1)^{y} C_{\sigma_{3}}\right]}{4}\right. \\
& \left.\log _{2} \frac{1-(-1)^{x+y} C_{\sigma_{2}}}{2}\right\} \\
& -\sum_{x, y=0,1}\left\{\frac{\left[1-(-1)^{x+y} C_{\sigma_{2}}\right] \cdot\left[1+(-1)^{y} C_{\sigma_{3}}\right]}{4}\right. \\
& \left.\log _{2} \frac{1+(-1)^{y} C_{\sigma_{3}}}{2}\right\} \\
& =-\frac{1-C_{\sigma_{2}}}{2} \log _{2} \frac{1-C_{\sigma_{2}}}{2}-\frac{1+C_{\sigma_{2}}}{2} \log _{2} \frac{1+C_{\sigma_{2}}}{2} \\
& -\frac{1-C_{\sigma_{3}}}{2} \log _{2} \frac{1-C_{\sigma_{3}}}{2}-\frac{1+C_{\sigma_{3}}}{2} \log _{2} \frac{1+C_{\sigma_{3}}}{2} \\
& =H_{b i n}\left(\frac{1+C_{\sigma_{2}}}{2}\right)+H_{b i n}\left(\frac{1+C_{\sigma_{3}}}{2}\right) \text {, }
\end{aligned}
$$

which is equal to the uncertainty of the observables $\sigma_{2}$ and $\sigma_{3}$ [Eq. (3)]. For other two SPMC conditions, the proof is similar.

\section{Expression of Eq. (10)}

Although a Bell-diagonal state under local amplitudedamping channel will no longer be of the Bell-diagonal type, it is still of " $\mathrm{X}$ " type

$$
\rho_{A B}(t)=\frac{1}{2}\left(\begin{array}{llll}
v_{+} & 0 & 0 & w_{-} \\
0 & v_{-} & w_{+} & 0 \\
0 & w_{+} & 1-v_{+} & 0 \\
w_{-} & 0 & 0 & 1-v_{-}
\end{array}\right),
$$

with $v_{ \pm}=e^{-\Gamma_{a d} t}\left(1 \pm C_{\sigma_{3}}\right) / 2$ and $w_{ \pm}=e^{-\Gamma_{a d} t / 2}\left(C_{\sigma_{1}} \pm\right.$ $\left.C_{\sigma_{2}}\right) / 2$. In general, we may employ projectors $\left\{B_{m}\right\}=$ $\left\{\cos \theta|0\rangle+e^{i \xi} \sin \theta|1\rangle, e^{-i \xi} \sin \theta|0\rangle-\cos \theta|1\rangle\right\}$ [1]. If $\left|w_{+}+w_{-}\right| \geq\left|w_{+}-w_{-}\right|$, i.e., $\left|C_{\sigma_{1}}\right| \geq\left|C_{\sigma_{2}}\right|$, the optimal measurement is either $\{(|0\rangle+|1\rangle) / \sqrt{2},(|0\rangle-$ $|1\rangle) / \sqrt{2}\}$, i.e., $\sigma_{x}$ operation or $\{|0\rangle,-|1\rangle\}$, i.e., $\sigma_{z}$ operation [2]. Therefore, the minimal missing information after local measurement can be expressed as $M=\min \left\{M_{x}, M_{z}\right\}$, where $M_{x}=H_{b i n}\left(\frac{1+u}{2}\right)$ with $u=\sqrt{e^{-\Gamma_{a d} t}\left[C_{\sigma_{1}}^{2}+2 \cosh \left(\Gamma_{a d} t\right)-2\right]}$ and $M_{z}=$ $\frac{H_{b i n}\left(v_{+}\right)+H_{b i n}\left(v_{-}\right)}{2}$. With $M$, the discord can be calculated straightforwardly. 


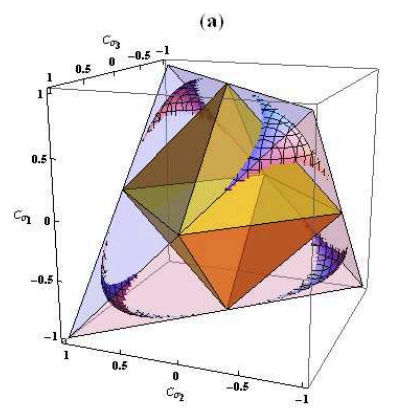

(b)

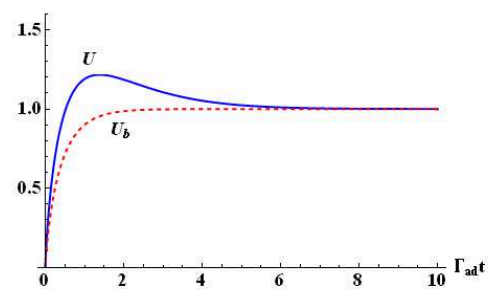

Figure.SM 4: (Color online) (a) The geometry of Bell-diagonal states. The yellow octahedron is the set of separable states while the four little blue tetrahedrons are set of entangled states. The four meshed curve surfaces correspond to the critical condition for the decrease (inside) or increase (outside) of uncertainty (lower bound) under local nonunitial amplitude-damping channel in the long-time limit. (b) A trivial example: The initial state prepared in $\left(C_{\sigma_{1}}, C_{\sigma_{2}}, C_{\sigma_{3}}\right)=(-1,1,1)$ will increase the uncertantiy (lower bound) in the long-time limit.

\section{The role of the initial state for the decrease of uncertainty under nonunital amplitude-damping channel}

Will all initial states prepared in Bell-diagonal type under the influence of local nonunital amplitude-damping noise decrease the uncertainty (lower bound) in the longtime limit? The answer is no. Actually, only if we prepare the initial Bell-diagonal state satisfying the condition $U_{b}(0)>U_{b}(\infty)$, will the phenomenon illustrated in the manuscript arise. To see more clearly, a geometry for the condition is depicted in Figure.SM(a): initial states inside the four meshed curve surfaces are those satisfying the condition. As a trivial example (for the uncertainty lower bound increasing under the noise), we consider one of the four Bell states $\left(C_{\sigma_{1}}, C_{\sigma_{2}}, C_{\sigma_{3}}\right)=(-1,1,1)$, i.e., the vertex of the tetrahedron, as the initial state. Figure.SM(b) shows that the uncertainty (lower bound) will increase in the long-time limit compared with the situation when no noise takes place.

\section{The proof of increasing uncertainty under local unital noisy channels}

Proof. Consider a bipartite system $\mathrm{AB}$ with dimension $d_{A B}=d_{A} \times d_{B}$. We first demonstrate that if the local noisy channel $\Lambda_{u}^{A}$ is unital, then the map $\mathcal{M}_{l u}=\left[\Lambda_{u}^{A} \otimes \mathbb{1}^{B}\right]$ is still unital. The proof is straightforward: $\mathcal{M}_{l u}\left(\frac{\mathbb{1}^{A B}}{d_{A B}}\right)=\sum_{\mu}\left(\kappa_{\mu} \otimes \mathbb{1}\right) \frac{\mathbb{1}^{A B}}{d_{A B}}\left(\kappa_{\mu} \otimes \mathbb{1}\right)^{\dagger}=$ $\sum_{\mu}\left(\kappa_{\mu} \frac{\mathbb{1}^{A}}{d_{A}} \kappa_{\mu}^{\dagger}\right) \otimes \frac{\mathbb{1}^{B}}{d_{B}}=\frac{\mathbb{1}^{A}}{d_{A}} \otimes \frac{\mathbb{1}^{B}}{d_{B}}=\frac{\mathbb{1}^{A B}}{d_{A B}}$.

Recalling $U_{b}=\log _{2} \frac{1}{c}+S(A \mid B)=\log _{2} \frac{1}{c}+S\left(\rho_{A B}\right)-$ $S\left(\rho_{B}\right)$. If the state is initially prepared in the maximally mixed subsystems, i.e., $\rho_{A}=\frac{\mathbb{1}^{A}}{d_{A}}\left(\rho_{B}=\frac{\mathbb{1}^{B}}{d_{B}}\right)$, then quantum memory $B$ free from noise will keep maximally mixed: $S\left(\rho_{B}\right) \equiv \log _{2} d_{B}$ [3]. Therefore $U_{b}$ is fully dependent on $S\left(\rho_{A B}\right)$ and our task now is to prove $S\left[\mathcal{M}_{l u}\left(\rho_{A B}\right)\right] \geq S\left(\rho_{A B}\right)$. The proof can be with the help of the monotonicity of the relative entropy for quantum maps: $S\left[\mathcal{M}\left(\rho_{A B}\right) \| \mathcal{M}\left(\frac{\mathbb{1}^{A B}}{d_{A B}}\right)\right] \leq S\left[\rho_{A B} \| \frac{\mathbb{1}^{A B}}{d_{A B}}\right][4]$. Then we have $-S\left[\mathcal{M}\left(\rho_{A B}\right)\right]-\operatorname{tr}\left[\mathcal{M}\left(\rho_{A B}\right) \log _{2} \mathcal{M}\left(\frac{\mathbb{1}^{A B}}{d_{A B}}\right)\right] \leq$ $-S\left(\rho_{A B}\right)-\operatorname{tr}\left[\rho_{A B} \log _{2} \frac{\mathbb{M}^{A B}}{d_{A B}}\right]$
$\log _{2} d_{A B} . \quad$ If $\mathcal{M}$ is unital, then $\operatorname{tr}\left[\mathcal{M}_{l u}\left(\rho_{A B}\right) \log _{2} \mathcal{M}_{l u}\left(\frac{\mathbb{1}^{A B}}{d_{A B}}\right)\right]=\operatorname{tr}\left[\mathcal{M}_{l u}\left(\rho_{A B}\right) \log _{2} \frac{\mathbb{1}^{A B}}{d_{A B}}\right]=$ $-\log _{2} d_{A B}$. Finally, we may obtain $S\left[\mathcal{M}_{l u}\left(\rho_{A B}\right)\right] \geq$ $S\left(\rho_{A B}\right)$, which implies that $U_{b}$ will not decrease under local unital noisy channels. However, this conclusion does not apply to local nonunital noisy channels.

* Electronic address: zhenyuxu@suda.edu.cn

$\dagger$ Electronic address: mangfeng@wipm.ac.cn

[1] H. Ollivier and W. H. Zurek, Phys. Rev. Lett. 88, 017901 (2001); L. Henderson and V. Vedral, J. Phys. A 34, 6899 (2001).

[2] M. Ali et al., Phys. Rev. A 81, 042105 (2010); Q. Chen et al., ibid. 84, 042313 (2011).

[3] M. A. Nielsen and I. L. Chuang, Quantum Computation and Quantum Information (Cambridge University Press, Cambridge, England, 2000).

[4] V. Vedral et al., Phys. Rev. Lett. 78, 2275 (1997); T. Rybár and M. Ziman, Phys. Rev. A 78, 052114 (2008); P. J. Coles et al., ibid. 83, 062338 (2011). 